\title{
Finding Positive Meaning in Negative Experiences Engages Ventral Striatal and Ventromedial Prefrontal Regions Associated with Reward Valuation
}

\author{
Bruce P. Doré ${ }^{1}$, Chelsea Boccagno ${ }^{1}$, Daisy Burr ${ }^{1}$, Alexa Hubbard ${ }^{2}$, Kan Long ${ }^{1}$, \\ Jochen Weber ${ }^{1}$, Yaakov Stern ${ }^{1}$, and Kevin N. Ochsner ${ }^{1}$
}

\begin{abstract}
Neuroimaging research has identified systems that facilitate minimizing negative emotion, but how the brain is able to transform the valence of an emotional response from negative to positive is unclear. Behavioral and psychophysiological studies suggest a distinction between minimizing reappraisal, which entails diminishing the arousal elicited by negative stimuli, and positive reappraisal, which instead changes the emotional valence of arousal from negative to positive. Here we show that successful minimizing reappraisal tracked with decreased activity in the amygdala, but successful positive reappraisal tracked with increased activity in regions involved
\end{abstract}

\section{INTRODUCTION}

Distressing life events are an unavoidable aspect of human experience. Many areas of research converge to demonstrate that how we respond to these events matters for our long-term mental and physical well-being (Gross, 2015; Folkman, 2008; Bonanno, 2004). Beyond simply dampening negative reactions to stressors, the ability to find positive meaning in potentially traumatic circumstances has been consistently identified as a protective factor against the harmful effects of stress and a critical pathway to resilience-the maintenance or enhancement of function in the face of potential trauma (Russo, Murrough, Han, Charney, \& Nestler, 2012; Helgeson, Reynolds, \& Tomich, 2006; Tugade \& Fredrickson, 2004). For example, behavioral studies show that expressing positive emotion when speaking about a recent bereavement predicts improved psychological functioning over several years (Bonanno \& Keltner, 1997) and finding benefits or life lessons in experiencing a heart attack predicts improvement in health and decreased risk for a subsequent attack (Affleck, Tennen, Croog, \& Levine, 1987).

Despite its clear importance, virtually nothing is known about the neural mechanisms that underlie the ability to

${ }^{1}$ Columbia University, ${ }^{2} \mathrm{New}$ York University in computing reward value, including the ventral striatum and ventromedial $\mathrm{pFC}$ (vmPFC). Moreover, positive reappraisal enhanced positive connectivity between vmPFC and amygdala, and individual differences in positive connectivity between vmPFC and amygdala, ventral striatum, dorsomedial pFC, and dorsolateral pFC predicted greater positive reappraisal success. These data broaden models of emotion regulation as quantitative dampening of negative emotion and identify activity in a network of brain valuation, arousal, and control regions as a neural basis for the ability to create positive meaning from negative experiences. find positive meaning and, in turn, generate positive emotion in the face of adversity. Addressing this gap in the literature builds our basic understanding of the neurobiology underlying human emotional capabilities and helps clarify the precise brain processes that may fail in psychopathology (Kring \& Sloan, 2009).

Focusing on the positive implications of a distressing experience can be understood as an instance of reappraisal, a strategy for emotion regulation that entails reframing the meaning of an emotional situation. Laboratory research has typically considered reappraisal as a uniform strategy, ignoring potentially crucial differences between different ways of reappraising (Buhle et al., 2014; Ochsner, Silvers, \& Buhle, 2012). In particular, a fundamental distinction can be made between minimizing reappraisal, which entails focusing on unemotional aspects of a negative experience to dampen negative feelings, and positive reappraisal, which entails focusing on positive aspects of a negative experience to enhance positive feelings (McRae \& Mauss, in press; McRae, Ciesielski, \& Gross, 2012).

Prior neuroimaging studies indicate that reappraisal of negative stimuli elicits increased activity in brain regions associated with domain-general cognitive control, including ventrolateral pFC (vlPFC), dorsolateral pFC (dlPFC), dorsomedial pFC (dmPFC), and lateral parietal cortex, alongside decreased activity in brain regions associated 
with emotional responding, including the amygdala (Buhle et al., 2014). However, converging lines of evidence suggest that positive reappraisal may engage distinct neural systems.

First, although early theories of amygdala function associated this region with negative emotions like fear (LeDoux, 1998), more recent work has shown that the amygdala responds more generally to material that is relevant to our goals, including stimuli that are positive, arousing, novel, ambiguous, or surprising (Cunningham \& Brosch, 2012; Whalen, 1998). Second, behavioral and psychophysiological studies have observed that, unlike minimizing reappraisal, positive reappraisal does not diminish arousal elicited by negative stimuli but instead changes the emotional valence of this arousal from negative to positive (McRae et al., 2012; Shiota \& Levenson, 2012). Third, neuroscience research has characterized a system of brain regions, including the ventral striatum (which includes nucleus accumbens) and ventromedial $\mathrm{pFC}$ (vmPFC) that are involved in computing reward value and thought to underlie positive emotional responding across a wide variety of contexts (Bartra, McGuire, \& Kable, 2013; Haber \& Knutson, 2010; Sabatinelli, Bradley, Lang, Costa, \& Versace, 2007; Mobbs, Greicius, AbdelAzim, Menon, \& Reiss, 2003).

To the extent that positive reappraisal successfully elicits positive emotion, it should not modulate the amygdala, but rather the ventral striatum and vmPFC regions associated with reward. Moreover, vmPFC and amygdala are reciprocally interconnected via dense white matter projections (Freese \& Amaral, 2009) and have been observed to show both negative (Johnstone, van Reekum, Urry, Kalin, \& Davidson, 2007) and positive patterns of coactivation (Erk et al., 2010; Banks, Eddy, Angstadt, Nathan, \& Phan, 2007) during emotion regulation. However, it is not yet clear what this coactivation reflects. Following theories of vmPFC as a region computing an integrative and contextually sensitive signal for positive value (Roy, Shohamy, \& Wager, 2012; Rangel \& Hare, 2010), it may be that positive connectivity between amygdala and vmPFC reflects the operation of a neural circuit supporting changes in the valence of affective arousal from negative to positive. If so, vmPFC should show enhanced positive connectivity with the amygdala during positive reappraisal.

Taken together, these separate lines of work suggest that positive reappraisal and minimizing reappraisal may show similarities in engagement of control-related prefrontal and parietal regions, but differences in the extent to which they modulate brain regions associated with affective arousal and positive value as well as the extent to which they rely on vmPFC to amygdala connectivity.

We used behavioral and fMRI methods to address these questions. In a preliminary behavioral analysis, we used affect ratings made in the fMRI scanner and text data we collected in a separate cohort of participants to examine the effects of these experimental conditions on emotional experience and the linguistic content of each type of reappraisal. In our neuroimaging study, we tested four hypotheses about the neural mechanisms of positive versus minimizing reappraisals. First, we hypothesized that positive and minimizing reappraisal would engage common regions of prefrontal and parietal cortex. Second, we hypothesized that positive reappraisal would modulate activity in the amygdala to a lesser extent than minimizing reappraisal. Third, we hypothesized that positive reappraisal would modulate activity in the ventral striatum and vmPFC to a greater extent than minimizing reappraisal. Finally, we hypothesized that successful positive reappraisal would rely on enhanced connectivity between the amygdala and the vmPFC.

\section{METHODS \\ Participants}

Participants were 20 adults (12 women, 8 men) recruited from the New York City area (mean age $=24.6, S D=4.5$ ) and screened to confirm that they could read and speak fluently in English, had normal or corrected-to-normal vision, had never been diagnosed with a psychiatric disorder, did not report current depressive symptoms (i.e., scored below 16 on the Center for Epidemiologic Studies Depression Scale), and had no conditions that contraindicated magnetic resonance imaging (MRI). A separate cohort of 20 adults (11 women, 9 men; mean age $=21.5$, $S D=3.1)$ completed a behavioral study in which they typed texts representing the content of their reappraisals and natural responses. All study procedures were approved by the Columbia University institutional review board.

\section{Image Acquisition}

Data were collected with a 3T MR750 (GE Healthcare Systems, Milwaukee, WI) magnet and a 32-channel RF head coil. Structural volumes were acquired using a high-resolution T1-weighted sagittal 3-D BRAVO sequence yielding $1 \mathrm{~mm}^{3}$ isotropic voxel size. Functional volumes were acquired using a T2*-sensitive EPI sequence with a repetition time of $2000 \mathrm{msec}$, an echo time of $25 \mathrm{msec}$, a $77^{\circ}$ flip angle, and a field of view of $19.2 \mathrm{~cm}$ consisting of 45 interleaved $3-\mathrm{mm}$ slices acquired parallel to the AC-PC axis. Four runs of 185 repetition times were collected. Each run began with 8 sec of fixation, and the corresponding four volumes were discarded.

\section{Task Design}

Participants completed an experimental task consisting of six functional runs. One half (three consecutive runs) of the experimental task was devoted to positive reappraisal (with positive reappraisal trials, negative image trials, and neutral image trials intermixed), and the other half (three consecutive runs) was devoted to minimizing 
reappraisal (with minimizing reappraisal trials, negative image trials, and neutral image trials intermixed). The order of positive and minimizing reappraisal was counterbalanced across participants. Each run consisted of 15 trials each: five negative image reappraisal trials, five negative image natural response trials, and five neutral image trials. The trial sequence, consisting of cue, image, ISI, rating period (counterbalanced order), and intertrial interval, is represented in Figure 1. Aversive images (mean valence $=$ 2.49; mean arousal $=5.71$ ) and neutral images (mean valence $=5.41$; mean arousal $=3.54$ ) were selected from the International Affective Picture System (Lang, Greenwald, Bradley, \& Hamm, 1993). Aversive images were counterbalanced to experimental condition across participants. Before scanning, participants completed experimenterguided training modules for both types of reappraisal, which included examples of acceptable positive and minimizing reappraisals and opportunities to practice. For positive reappraisal, participants were told to think about the depicted situations by focusing on potential positive aspects or outcomes, and for minimizing reappraisal, to think about potential neutral aspects or outcomes. For natural response trials, participants were told to view and think about the images as they normally would. Stimuli were presented with E-Prime 1.2 (Psychology Software Tools, Pittsburgh, PA). Participants made behavioral responses on a five-button response pad.

A separate cohort of participants completed a behavioral study in which they received identical training procedures, viewed a subset of the same images (38 of the 60 used in the MRI study; mean valence $=2.41$; mean arousal $=5.97$ ), and were asked to type for each image a short phrase or sentence representing the content of their natural response, minimizing reappraisal, or positive reappraisal.

\section{Behavioral Analysis}

Behavioral ratings of negative and positive affect tended to be negatively correlated within participants (mean $r=$ -.36 for natural response; mean $r=-.43$ for positive reappraisal; mean $r=-.29$ for minimizing reappraisal). These ratings were differenced (positive - negative) to yield the overall valence of reported experience on each trial of the task. Next, valence reports were averaged within participants and analyzed with planned $t$ tests to test hypotheses about effects of positive reappraisal and

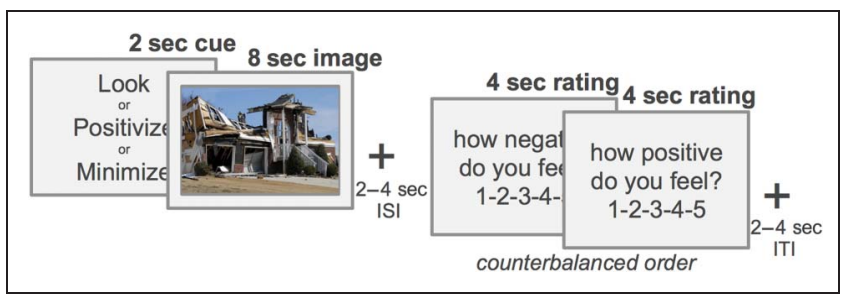

Figure 1. Trial sequence for the fMRI reappraisal task. minimizing reappraisal on the valence of affective experience. Ratings of negative and positive affect were also used to compute a positive reappraisal success score for each participant, reflecting a combined score for success in changing affective valence by down-regulating negative affect (mean Natural Response rating - mean Positive Reappraisal rating) and up-regulating positive affect (mean Positive Reappraisal rating - mean Natural Response rating).

Reappraisal and natural response texts (generated by a separate cohort) were processed with Linguistic Inquiry and Word Count (liwc.net), a prominent dictionary-based text analysis software, yielding proportions of words from Linguistic Inquiry and Word Count negative emotion (negemo) and positive emotion (posemo) categories used by participants on each trial. These text proportions were also averaged within participants and analyzed with planned $t$ tests. Finally, texts were also processed with a dictionary-free text analytic method (Fellows, 2014) to descriptively visualize relative frequencies of particular words across positive reappraisal, minimizing reappraisal, and natural response conditions.

\section{fMRI Analysis}

\section{Preprocessing/General Linear Model}

Data preprocessing was conducted with SPM8 (Wellcome Department of Cognitive Neurology, University College London) and consisted of slice-time correction, realignment, coregistration of functional and structural images, and normalization to the standard Montreal Neurological Institute brain by segmentation of the structural image and applying the parameters from this step during warping. Normalized images were interpolated to $3 \mathrm{~mm}^{3}$ voxels and smoothed with a 6-mm Gaussian kernel.

First-level (individual) general linear modelanalyses were implemented in NeuroElf v1.0 (neuroelf.net), using robust estimation via iteratively reweighted least squares (see Wager, Keller, Lacey, \& Jonides, 2005), to reduce the influence of time-series outliers (e.g., sudden head motion or other artifactual changes in signal intensity) on the estimated model parameters. Cue, stimulus, and response periods of each trial were modeled as boxcar functions convolved with the canonical hemodynamic response function. Separate stimulus regressors were entered for positive reappraisal, natural response (within positive reappraisal block), neutral images (within positive reappraisal block), minimizing reappraisal, natural response (within minimizing reappraisal block) conditions, and neutral images (within minimizing reappraisal block) as well as condition-centered parametric regressors for trial-bytrial reports of valence (i.e., positive affect rating minus negative affect rating) in each of these four conditions. Motion parameters and a high pass temporal filter for $128 \mathrm{sec}$ were added as regressors of no interest.

Second-level (group) random-effects analyses were implemented in NeuroElf v1.0. All activation peaks are 
reported in standard Montreal Neurological Institute space. ROIs for bilateral amygdala ( $\mathrm{L}-25,-2,-18$; R 27, -2, $-28 ; 5324 \mathrm{~mm}^{3}$ ) and nucleus accumbens ( $\mathrm{L}-9,11,-7$; R 9, 12, $-6 ; 1422 \mathrm{~mm}^{3}$ ) were defined anatomically, using volumes from the Harvard-Oxford probabilistic atlas (thresholded at $25 \%$ probability), and an ROI for vmPFC (peak 0, 46, $-6 ; 4860 \mathrm{~mm}^{3}$ ) was defined on the basis of a meta-analysis identifying brain regions carrying a monotonic, modality-independent signal for subjective reward value (shown in Figure 9 of Bartra et al., 2013). An additional anatomically and functionally constrained amygdala ROI was defined by computing the contrast of [natural response (both blocks)] > [neutral images (both blocks)] within the mask for bilateral amygdala at $p<.05$, identifying a cluster of 33 contiguous voxels within the left amygdala. This targeted ROI allowed us to test for modulation of the region of the amygdala that was responsive to the presentation of aversive images. For effect size estimation across entire ROIs, beta estimates were extracted, and means and standard errors were computed. For search analyses within ROIs, small-volume correction was applied to achieve a corrected $p$ value of $<.05$, using Gaussian random field theory to estimate the number of independent resolution elements in each ROI. For wholebrain analyses, cluster-extent thresholding was applied, using AlphaSim Monte Carlo simulation to achieve a whole-brain FWE rate corrected $p$ value of $<.05$, with a primary threshold of $p=.005$ (i.e., $z \approx 2.6$ ) and smoothness parameters $(9.7-11.4 \mathrm{~mm})$ estimated from the residuals of each statistical map to determine a minimum number of contiguous voxels, $k$ (from 103 to 142 for individual contrasts; 10 for a conjunction map).

\section{Functional Connectivity}

To examine changes in coactivation across brain regions, we conducted a psychophysiological interaction (PPI) analysis. As a seed, we used the region of vmPFC that parametrically tracked affect ratings for positive reappraisal to a greater extent than natural response and minimizing reappraisal trials, as identified in a whole- brain parametric analysis. For this PPI, regressors were entered for each experimental condition, the seed region time series, and interaction terms for the seed region time series and the experimental conditions. In a group-level test, we contrasted the PPI terms for the positive reappraisal condition over the natural response and minimizing reappraisal conditions (i.e., $[1-0.5-0.5])$ to identify regions of the brain that change in connectivity with vmPFC for positive reappraisal relative to the other task conditions. Next, we correlated this PPI map with success scores to identify regions for which connectivity was correlated with behavioral success in positive reappraisal.

\section{RESULTS}

\section{Manipulation Check: Positive Reappraisal and Minimizing Reappraisal Have Distinct Effects on Affective Experience and Linguistic Behavior}

First, we asked whether positive reappraisal, minimizing reappraisal, and natural responses were associated with differences in self-reported affect. As expected, relative to the natural response condition, when using minimizing reappraisal participants reported feeling less negative (reaching neutral valence), $t(19)=10.71, p<.0001$, and when using positive reappraisal participants reported feeling less negative and more positive (i.e., engendering positively valenced responses), $t(19)=18.16, p<.0001$ (see Figure 2A).

To provide insight into the content of reappraisals, in a separate cohort of participants we asked whether word use differed in brief typed texts representing the content of their natural responses, positive reappraisals, and minimizing reappraisals. We found an interaction of Condition (natural response, minimizing reappraisal, positive reappraisal) and Word category (negative emotion words, positive emotion words), $F(1,19)=57.87, p<$ .0001 , on proportion of emotion words used in these texts. Compared with natural response texts, we found that positive reappraisal texts had fewer negative emotion words, $t(19)=10.31, p<.0001$, and more positive
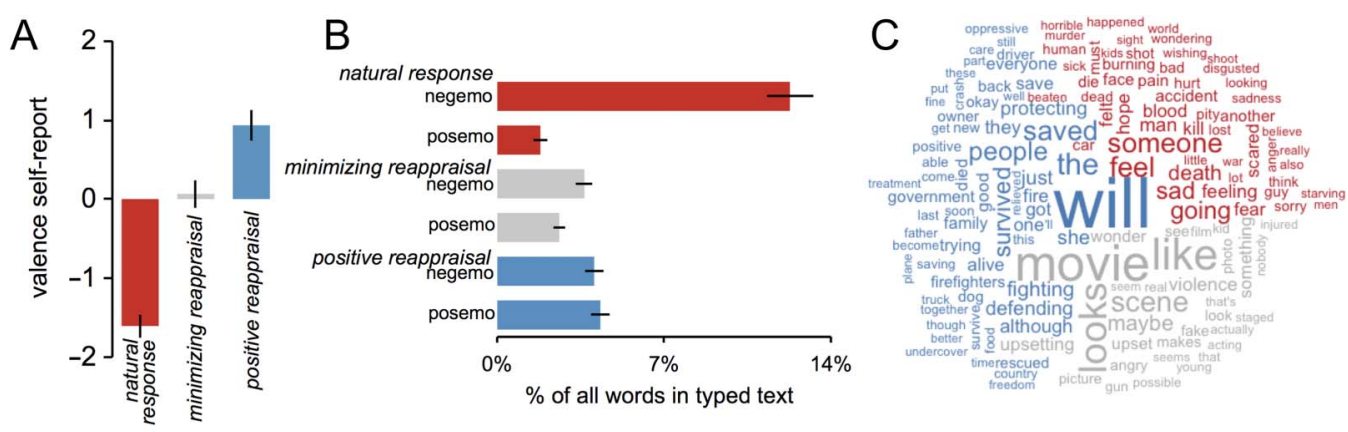

Figure 2. (A) Effects of experimental condition on self-reported valence of affective experience. (B) Effects of experimental condition on use of words from negative emotion (negemo) and positive emotion (posemo) categories. (C) Comparison cloud visualizing individual words that show the highest relative frequencies of use for natural response (red), minimizing reappraisal (gray), and positive reappraisal (blue) conditions. Means $\pm S E$. 


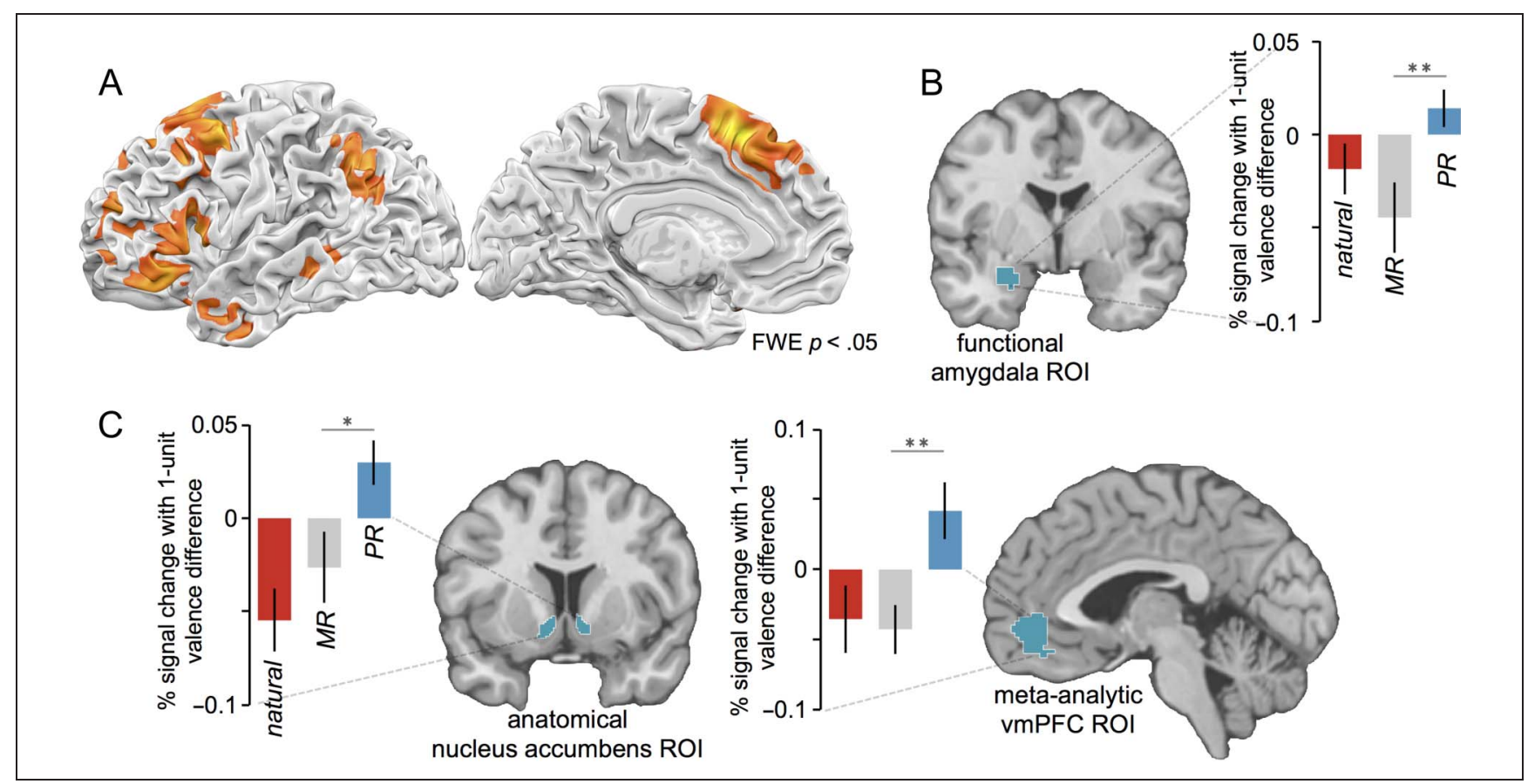

Figure 3. (A) Common regions of activation for minimizing and positive reappraisal, identified with a conjunction of reappraisal contrasts. (B) Mean betas $( \pm S E)$ extracted from an anatomically and functionally constrained amygdala ROI indicate more negative trial-to-trial modulation of the amygdala for minimizing reappraisal than positive reappraisal. (C) Mean betas ( $\pm S E$ ) extracted from an anatomical nucleus accumbens ROI and a meta-analytically defined vmPFC ROI indicate more positive trial-to-trial modulation for positive reappraisal than minimizing reappraisal.

emotion words, $t(19)=3.47, p=.002$. Similarly, compared with natural response texts, minimizing reappraisal texts had fewer negative emotion words, $t(19)=8.46, p<$ .0001 , and more positive emotion words at a trending level of significance, $t(19)=1.85, p=.08$. Finally, positive reappraisal texts had more positive emotion words than did minimizing reappraisal texts, $t(19)=2.96, p=.008$ (see Figure 2B). Next we used a data-driven text analytic method to identify words occurring at disproportionately high frequency for texts from a given condition. Words identified by this analysis are displayed in Figure 2C, a descriptive visualization of differences in word use across condition (text size reflects relative frequency for a given experimental condition relative to the other two).

\section{Hypothesis 1: Positive Reappraisal and Minimizing Reappraisal Engage Common Regions of Prefrontal and Parietal Cortex}

Next we turned to data from the fMRI study, focusing on the period of image presentation during which reappraisals are being implemented. To identify the regions of the brain associated with implementing positive reappraisal and minimizing reappraisal, we computed contrasts of both positive reappraisal $>$ natural response (within the positive reappraisal block) and minimizing reappraisal $>$ natural response (within the minimizing reappraisal block) conditions.

For positive reappraisal, we found activation in bilateral vlPFC (L -48, 27, -18; R 51, 24, -15), bilateral
dlPFC (L -33, 9, 51; R 18, 60, 30), bilateral dmPFC (L -3, 27, 33; R 6, 21, 33), left posterior parietal cortex (-45, $-57,30)$, bilateral temporal lobe ( $\mathrm{L}-57,-24,-15$; $\mathrm{R} 54,-12,-33)$, left dorsal caudate $(-15,9,12)$ and left putamen $(-15,12,0)$. For minimizing reappraisal, we found activation in left vlPFC $(-48,30,-9)$, left dlPFC $(-48,18,33)$, bilateral dmPFC (L $-3,27,36$; R 12, 18, $57)$, and left posterior parietal $(-54,-66,24)$. To identify neural mechanisms common to both types of reappraisal, we computed the conjunction of these two contrasts, revealing activity in left vlPFC $(-51,15,3)$, left dlPFC $(-39$, 21, 42), bilateral dmPFC (L -3, 27, 36; R 12, 18, 57), left posterior parietal cortex $(-51,-57,36)$, and both anterior $(-42,-3,-42)$ and posterior $(-60,-33,-6)$ regions of left lateral temporal cortex (see Figure 3A). Considering differences in the brain mechanisms associated with the state of implementing positive reappraisals and minimizing reappraisals, an interaction contrast of [positive reappraisal $>$ natural response] $>$ [minimizing reappraisal $>$ natural response] resulted in no significant whole brain clusters.

\section{Hypothesis 2: Minimizing Reappraisal Modulates Activity in the Amygdala More so than Positive Reappraisal}

Next we asked whether the two types of reappraisal differed in the extent to which they modulated activity in the amygdala from trial to trial. We examined betas for parametric valence regressors from an anatomically and 
functionally constrained ROI within the left amygdala, representing the region of the amygdala sensitive to the presentation of negative images. We found an interaction between Reappraisal tactic (positive reappraisal, minimizing reappraisal) and Trial type (reappraisal trial, natural response trial), $F(1,19)=5.55, p=.03$, for the parametric betas extracted from the amygdala. Critically, there was a difference, $t(19)=2.87, p=$ .01 , between the parametric effects for minimizing reappraisal (mean $b=-.044,95 \%$ CI $[-.081,-.007]$ ) and positive reappraisal (mean $b=.014,95 \% \mathrm{CI}$ $[-.005, .034])$, indicating that activity in this amygdala ROI was negatively modulated from trial to trial for minimizing reappraisal (such that less amygdala activity was apparent for trials for which less negatively valenced affect was reported), but not for positive reappraisal (see Figure 3B).

\section{Hypothesis 3: Positive Reappraisal Modulates Activity in the Ventral Striatum and vmPFC More so than Minimizing Reappraisal or Natural Responding}

Next we asked whether positive reappraisal differs from minimizing reappraisal and natural responding in the extent to which it modulates activity in regions of the brain associated with reward. For positive reappraisal, we observed positive parametric tracking of affective valence within the nucleus accumbens $(\mathrm{L}-9,21,-9$; $\mathrm{R} 12,15,-12$; SVC $p<.05)$ and vmPFC $(6,33,-15$; SVC $p<.05)$ ROIs, such that greater activity was observed in these regions for trials where participants reported greater positive affect.

Extracting average parametric betas from bilateral nucleus accumbens revealed an interaction between Reappraisal tactic and Trial type, $F(1,19)=10.48, p=$ .004 . Critically, there was a difference, $t(19)=2.63, p=$ .02 , between parametric effects for positive reappraisal (mean $b=.030,95 \%$ CI $[.006, .053]$ ) versus minimizing reappraisal (mean $b=-.026,95 \%$ CI $[-.063, .011]$ ) and a difference, $t(19)=4.55, p=.0002$, between para- metric effects for positive reappraisal versus the intermixed natural response condition (mean $b=-.055$, 95\% CI [ $-.088,-.022]$; see Figure 3C). Extracting average parametric betas from the vmPFC revealed an interaction between Reappraisal tactic and Trial type, $F(1,19)=7.67$, $p=.01$. Critically, there was a difference, $t(19)=3.43$, $p=.003$, between parametric effects for positive reappraisal (mean $b=.042,95 \%$ CI $[.002, .081]$ ) versus minimizing reappraisal (mean $\beta=-.043,95 \%$ CI $[-.077$, $-.001]$ ) and a difference, $t(19)=2.60, p=.02$, between parametric effects for positive reappraisal versus the intermixed natural response condition (mean $b=-.035,95 \%$ CI $[-.083, .012]$; see Figure 3C).

To follow up on these targeted ROI-based analyses, we computed a whole-brain contrast of parametric maps to identify regions, across the entire brain, that linearly tracked affective valence ratings more for the positive reappraisal condition than for the minimizing reappraisal or intermixed natural response conditions (positive reappraisal $>$ minimizing reappraisal + natural response). Because stimuli were counterbalanced across conditions, this analysis identified regions that tracked affect differentially as a result of the manipulation of experimental condition rather than as a result of stimulus factors (like trial-to-trial variation in image intensity). This analysis revealed significantly more positive parametric tracking of affect for positive reappraisal (compared with minimizing reappraisal and natural response conditions) within bilateral ventral striatum (L 12, 18, -6; R -12, 21, -9) and a subgenual region of right vmPFC $(9,27,-15$; see Figure 4) and no other regions. In a follow-up analysis, we asked what regions of the brain showed a full crossover interaction of parametric effects [positive reappraisal intermixed natural response $>$ minimizing reappraisal intermixed natural response]-similarly, this revealed activity in ventral striatum ( $\mathrm{L}-9,18,-9 ; \mathrm{R} \mathrm{12,18,-6)}$ and $\operatorname{vmPFC}(3,21,-9)$ but no other regions. These results indicate that ventral striatal and vmPFC regions of the brain positively track reported affect for the positive reappraisal condition to a greater extent than for the minimizing reappraisal and natural response conditions.

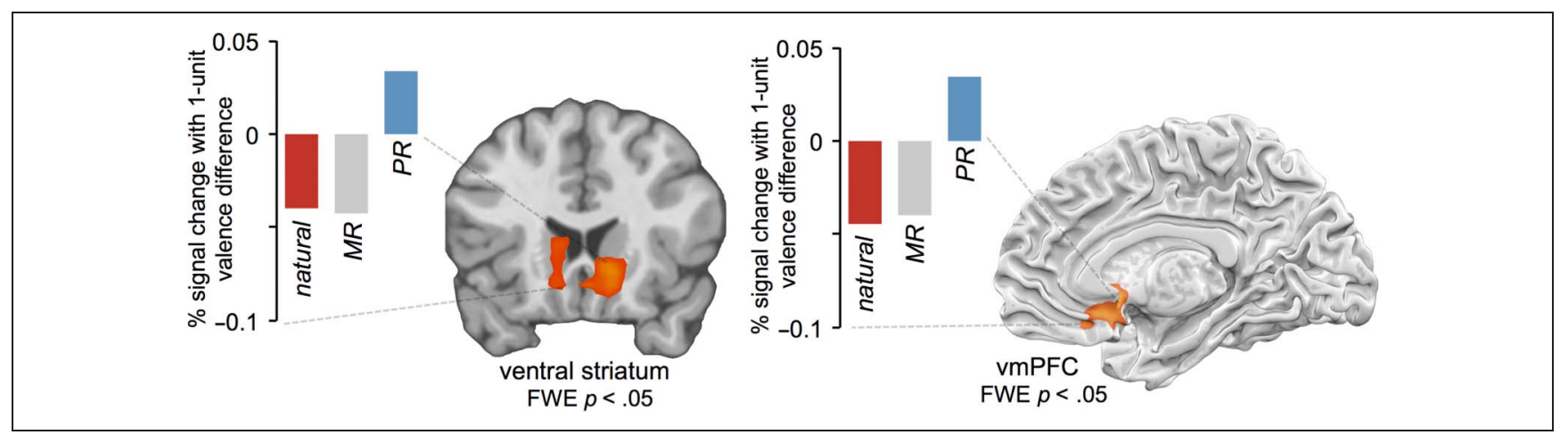

Figure 4. Regions tracking trial-to-trial affect for positive reappraisal condition more so than minimizing reappraisal or natural response, identified with a whole-brain contrast of parametric maps corrected at FWE $p<.05$. Mean betas shown for descriptive visualization-because these betas were derived from clusters derived from a whole-brain search, we omit error bars and significance tests. 


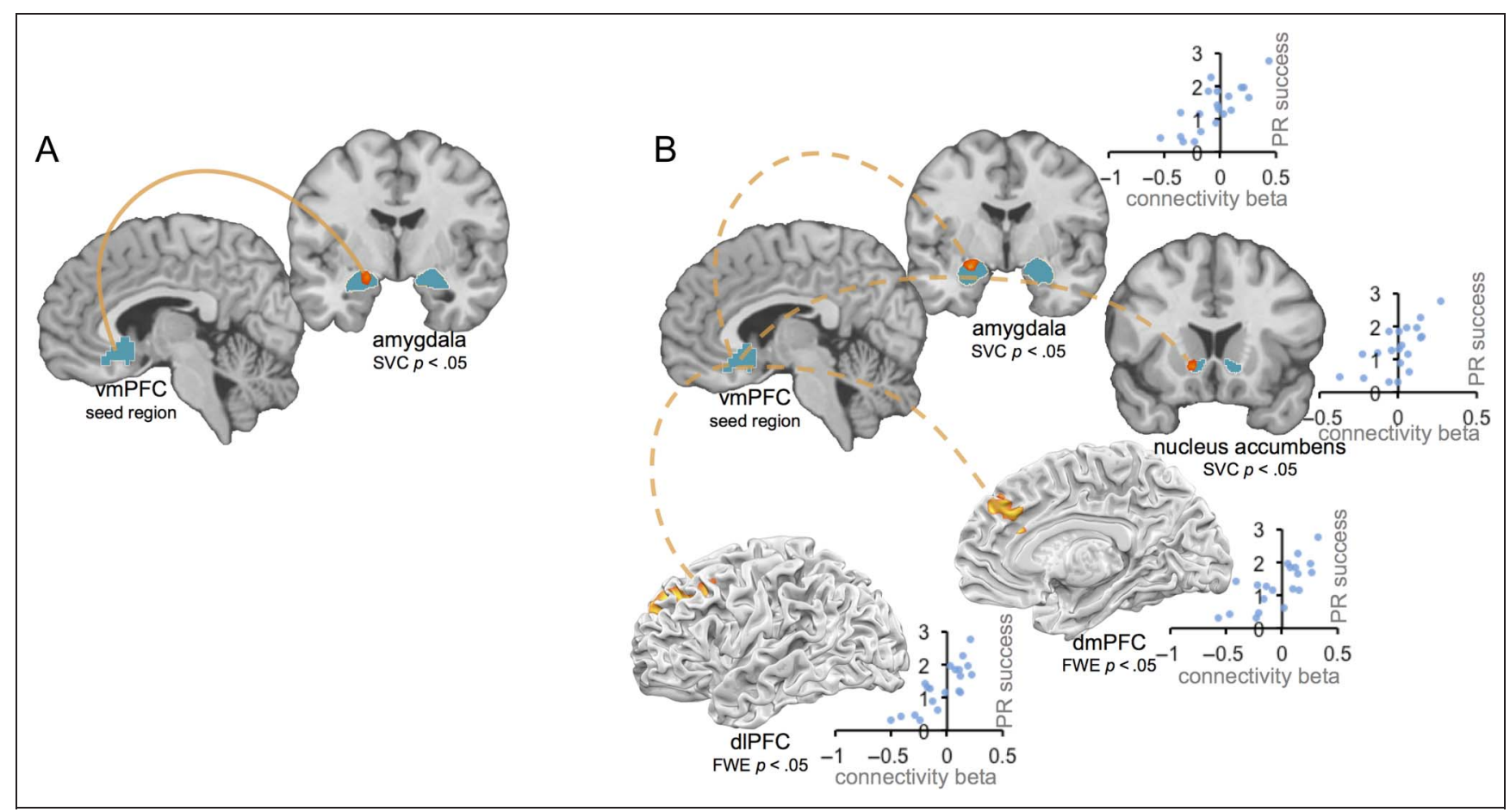

Figure 5. (A) Connectivity between vmPFC seed region and amygdala is enhanced for positive reappraisal, relative to natural response condition. (B) More positive connectivity between vmPFC seed region and amygdala, nucleus accumbens, dmPFC, and dlPFC regions predicts greater positive reappraisal success. Activations within amygdala and ventral striatum ROIs displayed at $p<.005$ uncorrected, and positive reappraisal success by connectivity beta scatterplots shown for descriptive visualization.

\section{Hypothesis 4a: Positive Reappraisal Enhances Positive Connectivity between vmPFC and Amygdala}

Hypothesizing that positive reappraisal relies on enhanced connectivity between the vmPFC and amygdala, we next conducted analyses that probed patterns of functional coactivation across the brain. In a functional connectivity (PPI) analysis, we used the region of the vmPFC identified in the contrast of parametric maps above (representing the vmPFC region tracking positive affect for positive reappraisal) as a seed and asked what regions of the brain show enhanced coactivation with this vmPFC seed for positive reappraisal relative to minimizing reappraisal and natural response trials. Consistent with our hypothesis, we found that vmPFC showed enhanced connectivity during positive reappraisal with left amygdala (peak $-18,-6,-15$; SVC $p<.05$; see Figure $5 \mathrm{~A}$ ). In a corresponding whole-brain analysis, no regions showed increased connectivity at significant or trend-level thresholds.

\section{Hypothesis 4b: More Positive vmPFC Connectivity with Amygdala, Striatum, dmPFC, and dlPFC Correlates with Positive Reappraisal Success}

Lastly, we conducted a follow-up analysis to ask whether differences in connectivity between vmPFC and other brain regions underlie person-to-person variability in positive reappraisal success. To do this, we computed a map reflecting, for each voxel, the correlation between each participant's vmPFC connectivity beta (for positive reappraisal vs. minimizing reappraisal and natural response trial types) and his or her positive reappraisal success score (reflecting success in up-regulating positive affect and down-regulating negative affect on positive reappraisal trials). We first examined a priori ROIs for amygdala and nucleus accumbens and found that person-to-person differences in positive reappraisal success were correlated with the extent to which vmPFC showed positive connectivity with bilateral amygdala $(\mathrm{L}-21,-3$, -12 ; R 18, $-9,-12$; SVC $p<.05)$ and bilateral nucleus accumbens (L $-12,15,-6$; R 18, 9, -12 ; SVC $p<.05$ ). Next we did a corresponding whole-brain analysis and found that person-to-person differences in positive reappraisal success also were correlated with connectivity betas for dmPFC $(-3,36,33)$ and left dlPFC $(-30,30$, 36; see Figure 5B). These analyses indicate that personto-person differences in connectivity between the vmPFC and brain regions associated with affective arousal, positive value, and cognitive control were correlated with positive reappraisal success.

\section{DISCUSSION}

A primary form of resilient coping involves finding positive meaning in negative life experiences. Here we performed the first test of the neural systems underlying 
this ability. Behaviorally, we found that positive reappraisal increased positive emotion and changed use of valenced linguistic content to a greater extent than did minimizing reappraisal. With fMRI, we found that successful minimizing reappraisal decreased activity in the amygdala, but successful positive reappraisal increased activity in ventral striatum and vmPFC regions associated with reward and positive affect such that activity in these regions tracked reports of more positive emotional experience. Finally, we found that that positive reappraisal was associated with enhanced connectivity between vmPFC and amygdala and person-to-person differences in connectivity between vmPFC and amygdala, ventral striatum, dmPFC, and dlPFC predicted overall success in using positive reappraisal.

\section{Implications for Neural Models of Emotion Regulation}

Where brain models of emotion regulation have previously highlighted the importance of interacting brain systems for top-down control and bottom-up generation of emotion, the results of this study extend these models in three ways. First, our results indicate a specific role for ventral striatal and vmPFC regions as a mechanism specific to positive reappraisal and not minimizing reappraisal or simply looking at negative images. Previous studies have not been designed to detect this pattern and may have failed to do so because they used reappraisal instructions that emphasized minimizing reappraisal (or did not clearly distinguish between minimizing and positive reappraisal) or because they applied a contrast-based analytic approach, rather than using parametric analyses to systematically relate reports of affective experience to underlying brain systems.

Second, brain models of emotion have highlighted that regulatory strategies can bring about decreased activity in the amygdala, concluding that diminished activity in this region reflects a decrease in negative emotion (see Denny, Ochsner, Weber, \& Wager, 2014; Ohira et al., 2006). However, our data suggest that modulation of the amygdala is not the only brain indicator of successful regulation of negative emotion. Using positive reappraisal to enhance the positive meaning of a stimulus maintains motivational relevance and affective arousal but shifts experienced emotional valence from negative to positive (McRae et al., 2012; Shiota \& Levenson, 2012). Our data identify engagement of ventral striatum and vmPFC reward regions and enhanced $\mathrm{vmPFC}$-amygdala positive coupling as neural mechanisms that underpin this shift in valence.

Third, the present data shed new light on models of emotion regulation and self-regulation more generally, positing that prefrontal control systems act in opposition to systems involved in emotion generation. On such views, increasing activity in one system, in the vmPFC, for example, down-regulates activity in regions of the other system, such as the amygdala (e.g., Diekhof, Geier,
Falkai, \& Gruber, 2011; Schiller \& Delgado, 2010). However, we did not observe that vmPFC down-regulates activity in the amygdala during positive reappraisal but rather that vmPFC and amygdala showed enhanced positive coupling, and the extent of this coupling was positively correlated with successfully changing one's emotional response from negative to positive. Thus, our data align better with theories describing the vmPFC as a nexus point that integrates information from cortical and subcortical brain regions (Roy et al., 2012; Rangel \& Hare, 2010). Beyond the amygdala, our data suggest that positive reappraisal success relies on vmPFC connectivity with a suite of regions associated with affective arousal, cognitive control, and positive value (see Barrett \& Satpute, 2013).

\section{Implications for the Study of Healthy and Clinical Variability in Affective Experience}

Our findings also raise novel questions for understanding clinical and subclinical variability in affective processes. Previous work suggests that emotion regulation ability can moderate the relationship between life stressors and depressive symptoms (Troy, Wilhelm, Shallcross, \& Mauss, 2010), that regulation of positive affect may reflect a transdiagnostic mechanism of emotional disturbance (Carl, Soskin, Kerns, \& Barlow, 2013), and that people suffering from depression show reduced ability to sustain affective responses to positive stimuli (Heller et al., 2009). We found that individual differences in positive reappraisal success related to functional connectivity of vmPFC, with dlPFC, dmPFC, amygdala, and ventral striatum, suggesting that these regions form a functional network that supports the ability to generate positive emotion in the face of negative stimuli.

A crucial future direction will be to connect models of the brain mechanisms underlying positive meaning to the broader literature on resilience and recovery from stressful life events. Although everyone experiences stressors, how we respond to them can range from protracted disability to functional maintenance and growth. Positive reappraisal ability, supported by vmPFC connectivity with amygdala, ventral striatum, and pFC, could be one factor that underlies such variability. Future work that integrates models of genetic, environmental, and neural underpinnings of variable responses to stress may help us understand where resilience comes from and how to optimize it (see Russo et al., 2012; Bonanno, 2004). Importantly, future studies of this kind will benefit from larger sample sizes, enabling more precise estimation of withinand (especially) between-person brain-behavior relationships and detection of smaller magnitude effects.

\section{Conclusion}

When life takes a turn for the worse, we cannot only minimize our negative reactions but fundamentally 
transform them to experience positive emotions like hope, gratitude, and love. Although negativity-minimizing and positivity-enhancing forms of emotion regulation are similar in that they rely on brain systems for controlled processing, they are distinct in that minimizing reappraisal modulates brain systems associated with affective arousal, whereas positive reappraisal modulates brain systems associated with reward value. Here we describe these distinct pathways to regulatory success and consider their implications for our broader understanding of emotion and its regulation. We hope that future work will expand on our approach to uncover the basic biological mechanisms that underlie our ability to respond flexibly and adaptively to the emotional challenges of everyday life.

\section{Acknowledgments}

We thank Ehjeen Kim for assistance with data collection; Laura Braunstein, Noam Zerubavel, Michael Gilead, Bryan Denny, and the rest of the Columbia SCAN lab for guidance and helpful discussion of this work; NIA grant R01AG043463-01 and Conte grant PAR-11-126 for support to K. O., and NIA grant R01AG026158-5 to Y. S. B. P. D. and K. N. O. designed the research. B. P. D., C. B., D. B., A. H., and K. L., collected the data. B. P. D. and J. W. analyzed the data. B. P. D. wrote the manuscript with critical contributions from K. N. O., J. W., and Y. S.

Reprint requests should be sent to Bruce P. Doré, Department of Psychology, Columbia University, Schermerhorn Hall, New York, NY 10027, or via e-mail: brucedore@gmail.com.

\section{REFERENCES}

Affleck, G., Tennen, H., Croog, S., \& Levine, S. (1987). Causal attribution, perceived benefits, and morbidity after a heart attack: An 8-year study. Journal of Consulting and Clinical Psychology, 55, 29-35.

Banks, S. J., Eddy, K. T., Angstadt, M., Nathan, P. J., \& Phan, K. L. (2007). Amygdala-frontal connectivity during emotion regulation. Social Cognitive and Affective Neuroscience, 2, 303-312.

Barrett, L. F., \& Satpute, A. B. (2013). Large-scale brain networks in affective and social neuroscience: Towards an integrative functional architecture of the brain. Current Opinion in Neurobiology, 23, 361-372.

Bartra, O., McGuire, J. T., \& Kable, J. W. (2013). The valuation system: A coordinate-based meta-analysis of BOLD fMRI experiments examining neural correlates of subjective value. Neuroimage, 76, 412-427.

Bonanno, G. A. (2004). Loss, trauma and human resilience: Conceptual and empirical connections and separateness. American Psychologist, 59, 20-28.

Bonanno, G. A., \& Keltner, D. (1997). Facial expressions of emotion and the course of conjugal bereavement. Journal of Abnormal Psychology, 106, 126-137.

Buhle, J. T., Silvers, J. A., Wager, T. D., Lopez, R., Onyemekwu, C., Kober, H., et al. (2014). Cognitive reappraisal of emotion: A meta-analysis of human neuroimaging studies. Cerebral Cortex, 24, 2981-2990.

Carl, J. R., Soskin, D. P., Kerns, C., \& Barlow, D. H. (2013). Positive emotion regulation in emotional disorders: A theoretical review. Clinical Psychology Review, 33, 343-360.
Cunningham, W. A., \& Brosch, T. (2012). Motivational salience amygdala tuning from traits, needs, values, and goals. Current Directions in Psychological Science, 21, 54-59.

Denny, B. T., Ochsner, K. N., Weber, J., \& Wager, T. D. (2014). Anticipatory brain activity predicts the success or failure of subsequent emotion regulation. Social Cognitive and Affective Neuroscience, 9, 403-411.

Diekhof, E. K., Geier, K., Falkai, P., \& Gruber, O. (2011). Fear is only as deep as the mind allows: A coordinate-based meta-analysis of neuroimaging studies on the regulation of negative affect. Neuroimage, 58, 275-285.

Erk, S., Mikschl, A., Stier, S., Ciaramidaro, A., Gapp, V., Weber, B., et al. (2010). Acute and sustained effects of cognitive emotion regulation in major depression. Journal of Neuroscience, 30, 15726-15734.

Fellow, I. (2013). Wordcloud: Word Clouds, R package version 2.4. URL: http://CRAN.R-project.org/package=wordcloud.

Folkman, S. (2008). The case for positive emotions in the stress process. Anxiety, Stress, and Coping, 21, 3-14.

Freese, J. L., \& Amaral, D. G. (2009). Neuroanatomy of the primate amygdala. In P. J. Whalen \& E. A. Phelps (Eds.), The buman amygdala. New York: Guilford Press.

Gross, J. J. (2015). The extended process model of emotion regulation: Elaborations, applications, and future directions. Psychological Inquiry, 26, 130-137.

Haber, S. N., \& Knutson, B. (2010). The reward circuit: Linking primate anatomy and human imaging. Neuropsychopharmacology, 35, 4-26.

Helgeson, V. S., Reynolds, K. A., \& Tomich, P. L. (2006). A meta-analytic review of benefit finding and growth. Journal of Consulting and Clinical Psychology, 74, 797-816.

Heller, A. S., Johnstone, T., Shackman, A. J., Light, S. N., Peterson, M. J., Kolden, G. G., et al. (2009). Reduced capacity to sustain positive emotion in major depression reflects diminished maintenance of fronto-striatal brain activation. Proceedings of the National Academy of Sciences, U.S.A., 106, 22445-22450.

Johnstone, T., van Reekum, C. M., Urry, H. L., Kalin, N. H., \& Davidson, R. J. (2007). Failure to regulate: Counterproductive recruitment of top-down prefrontal-subcortical circuitry in major depression. Journal of Neuroscience, 27, 8877-8884.

Kring, A. M., \& Sloan, D. M. (Eds.) (2009). Emotion regulation and psychopathology: A transdiagnostic approach to etiology and treatment. New York: Guilford Press.

Lang, P. J., Greenwald, M. K., Bradley, M. M., \& Hamm, A. O. (1993). Looking at pictures: Affective, facial, visceral, and behavioral reactions. Psychophysiology, 30, 261-273.

LeDoux, J. (1998). Fear and the brain: Where have we been, and where are we going?. Biological Psychiatry, 44, 1229-1238.

McRae, K., Ciesielski, B., \& Gross, J. J. (2012). Unpacking cognitive reappraisal: Goals, tactics, and outcomes. Emotion, 12, 250-255.

McRae, K., \& Mauss, I. B. (in press). Increasing positive emotion in negative contexts: Emotional consequences neural correlates and implications for resilience. In J. Green, I. Morrison, \& M. Seligman (Eds.), Positive neuroscience. Oxford: Oxford University Press.

Mobbs, D., Greicius, M. D., Abdel-Azim, E., Menon, V., \& Reiss, A. L. (2003). Humor modulates the mesolimbic reward centers. Neuron, 40, 1041-1048.

Ochsner, K. N., Silvers, J. A., \& Buhle, J. T. (2012). Functional imaging studies of emotion regulation: A synthetic review and evolving model of the cognitive control of emotion. Annals of the New York Academy of Sciences, 1251, E1-E24.

Ohira, H., Nomura, M., Ichikawa, N., Isowa, T., Iidaka, T., Sato, A., et al. (2006). Association of neural and physiological 
responses during voluntary emotion suppression

Neuroimage, 29, 721-733.

Rangel, A., \& Hare, T. (2010). Neural computations associated with goal-directed choice. Current Opinion in Neurobiology, 20, 262-270.

Roy, M., Shohamy, D., \& Wager, T. D. (2012). Ventromedial prefrontal-subcortical systems and the generation of affective meaning. Trends in Cognitive Sciences, 16, 147-156.

Russo, S. J., Murrough, J. W., Han, M. H., Charney, D. S., \& Nestler, E. J. (2012). Neurobiology of resilience. Nature Neuroscience, 15, 1475-1484.

Sabatinelli, D., Bradley, M. M., Lang, P. J., Costa, V. D., \& Versace, F. (2007). Pleasure rather than salience activates human nucleus accumbens and medial prefrontal cortex. Journal of Neurophysiology, 98, 1374-1379.

Schiller, D., \& Delgado, M. R. (2010). Overlapping neural systems mediating extinction, reversal and regulation of fear. Trends in Cognitive Sciences, 14, 268-276.
Shiota, M. N., \& Levenson, R. W. (2012). Turn down the volume or change the channel? Emotional effects of detached versus positive reappraisal. Journal of Personality and Social Psychology, 103, 416-429.

Troy, A. S., Wilhelm, F. H., Shallcross, A. J., \& Mauss, I. B. (2010). Seeing the silver lining: Cognitive reappraisal ability moderates the relationship between stress and depressive symptoms. Emotion, 10, 783.

Tugade, M. M., \& Fredrickson, B. L. (2004). Resilient individuals use positive emotions to bounce back from negative emotional experiences. Journal of Personality and Social Psychology, 86, 320-333.

Wager, T. D., Keller, M. C., Lacey, S. C., \& Jonides, J. (2005). Increased sensitivity in neuroimaging analyses using robust regression. Neuroimage, 26, 99-113.

Whalen, P. J. (1998). Fear, vigilance, and ambiguity: Initial neuroimaging studies of the human amygdala. Current Directions in Psychological Science, 7, 177-188. 